Proceedings of the International School and Conference on Optics and Optical Materials, ISCOM07, Belgrade, Serbia, September 3-7, 2007

\title{
Dancing Light: Counterpropagating Beams in Photorefractive Crystals
}

\author{
M.R. Belića ${ }^{a *}$, M.S. Petrović ${ }^{b}$, D.M. Jović ${ }^{b}$, A.I. Strinić ${ }^{b}$, \\ D.D. Arsenović ${ }^{b}$, S. PrVanović ${ }^{b}$, R.D. Jovanović $b$ \\ AND N.Z. Petrovića \\ ${ }^{a}$ Texas A \& M University at Qatar, P.O. Box 5825, Doha, Qatar \\ ${ }^{b}$ Institute of Physics, P.O. Box 57, Belgrade, Serbia
}

\begin{abstract}
A review of work on the dynamical behavior of counterpropagating incoherent laser beams in photorefractive crystals is presented. Numerical study of counterpropagating beams of different type is carried out, in both space and time, using an appropriate theoretical model. The development of patterns in broad hyper-Gaussian counterpropagating beams in saturable Kerr-like media is investigated, by varying the width of beams. Rotational properties of counterpropagating mutually incoherent self-trapped vortex beams in optically induced fixed photonic lattices are also investigated numerically. One of the fundamental quantum mechanical phenomena is observed for the counterpropagating beams in photonic lattices, the tunneling of light from the first to the higher-order bands of the lattice band gap spectrum. The transfer of angular momentum from vortex beams to optically induced photonic lattices is also demonstrated. For the interacting beams it is found that the sum of angular momenta of counterpropagating components is not a conserved quantity, but the difference is. In the fixed lattices there is always a considerable loss of angular momentum.
\end{abstract}

PACS numbers: 42.65.Tg, 42.65.Sf

\section{Introduction}

Owing to saturable, nonlinear and nonlocal response, and significant applicative potential, counterpropagating $(\mathrm{CP})$ beams in photorefractive $(\mathrm{PR})$ media have commanded considerable interest in recent years [1]. In a series of articles [2] we investigated the dynamics of mutually incoherent $\mathrm{CP}$ laser beams in local isotropic saturable PR crystals, both in time and in 3 spatial dimensions, using an appropriately developed theoretical model and a numerical procedure based on the

*corresponding author; e-mail: milivoj.belic@qatar.tamu.edu 
split-step beam propagation method. Theory capable of capturing such dynamics is introduced and linear stability analysis (LSA) performed. Numerical simulations are found in qualitative agreement with the experiment and LSA [3]. Spontaneous symmetry breaking of head-on Gaussian beams is demonstrated as the coupling strength is increased, resulting in the splitup transition of CP components. The development of patterns in broad hyper-Gaussian CP beams in saturable Kerr-like media is investigated, by varying the width of beams. Owing to slow quasi-steady-state changes in the system during time evolution, the transverse characteristics of beam distributions, determined by LSA, are found to persist for long in the transient evolution of $\mathrm{CP}$ beams. In the case of $\mathrm{CP}$ vortices, discrete diffraction, beam filamentation, as well as increased stability of the central vortex ring are displayed.

Rotational properties of CP mutually incoherent self-trapped vortex beams in optically induced fixed photonic lattices are also investigated numerically. The rotational dynamics in these systems is defined by the transport through tunneling between lattice sites. Various properties of CP mutually incoherent self-trapped beams in optically induced circular photonic lattices with ring defects are investigated numerically. Using a modified Petviashvili method, several novel solitonic structures are found. For CP beams in optically induced photonic lattices, one of the fundamental quantum mechanical phenomena is observed, the Zener tunneling of light from the first to the higher-order bands of the lattice band-gap spectrum. The existence of other phenomena characteristic of solid state physics, such as Bloch oscillations and Anderson localization [4] are demonstrated for the propagation of $\mathrm{CP}$ beams in optically induced photonic lattices.

The conservation of angular momentum of mutually incoherent copropagating $(\mathrm{CO})$ and counterpropagating lattice and vortex beams in saturable Kerr-like nonlinear media is discussed [5]. It is shown that the total angular momentum of $\mathrm{CO}$ beams is conserved, whereas that of $\mathrm{CP}$ beams is not. The difference of angular momenta of CP beams is conserved, not their sum. The transfer of angular momentum from the vortex beams to the optically induced photonic lattices is demonstrated. In the presence of optically induced lattices it is found that the total angular momentum is nearly conserved if the lattice beams are interacting with the $\mathrm{CP}$ vortices, and that it is not if the lattice beams are fixed. In the fixed lattices there is always a considerable loss of angular momentum.

We present here some of the typical examples involving these phenomena and add new results.

\section{The model}

The behavior of $\mathrm{CP}$ beams in photonic lattices is described by a time-dependent model for the formation of self-trapped CP optical beams, based on the theory of PR effect. The model consists of wave equations in the paraxial approximation for the propagation of $\mathrm{CP}$ beams and a relaxation equation for the 
generation of the space charge field in the crystal. Even though strictly speaking the PR nonlinearity is anisotropic and nonlocal, we choose to utilize a local isotropic saturable Kerr-like approximation. Qualitatively the same behavior is expected, at considerable savings in the computer memory and processing time. The model equations in the computational space are of the form [2]:

$$
\begin{aligned}
& \mathrm{i} \partial_{z} F=-\Delta F+\Gamma E F, \quad-\mathrm{i} \partial_{z} B=-\Delta B+\Gamma E B, \\
& \tau \partial_{t} E+E=-\frac{I+I_{\mathrm{g}}}{1+I+I_{\mathrm{g}}},
\end{aligned}
$$

where $F$ and $B$ are the forward and the backward propagating beam envelopes, $\Delta$ is the transverse Laplacian, $\Gamma$ is the dimensionless coupling constant, $\tau$ is the relaxation time of the crystal, and $E$ the homogeneous part of the space charge field. The change in the index of refraction of the medium is proportional to this field. The quantity $I=|F|^{2}+|B|^{2}$ is the total laser light intensity, measured in units of the background intensity. $I_{\mathrm{g}}$ is the transverse intensity distribution of the optically induced lattice array, which is formed by positioning Gaussian beams at the sites of the lattice. $I_{\mathrm{g}}$ is normalized by the dark irradiance $I_{\mathrm{d}}$. Transverse coordinates are scaled by the typical FWHM of beams, and the longitudinal coordinate by the corresponding diffraction length $L_{\mathrm{D}}$. The propagation equations are solved numerically, concurrently with the temporal equation, in the manner described in Ref. [2].

\section{Numerical results and discussion}

We first present the formation of various patterns in broad hyper-Gaussian CP beams of equal input intensity (Fig. 1). With the increasing value of the control parameter $A \Gamma L$, where $A=\left|F_{0}\right|^{2} /\left(1+\left|F_{0}\right|^{2}+\left|B_{L}\right|^{2}\right)^{2}$ and $L$ is the crystal thickness, a more complex behavior is observed. As the width of the CP beams is also increased, very rich dynamics at the exiting faces of the crystal is observed. For FWHM $=20 \mu \mathrm{m}$ we see a rotating displaced soliton, after a splitup transition. When FWHM is $40 \mu \mathrm{m}$, we see a tripole, and after that regular (tripole, quadrupole, ... ) oscillating structures. This indicates a Hopf bifurcation happening in the system. Similar situation is observed for FWHM $=60 \mu \mathrm{m}$, but with no oscillations after the transition. After a very short appearance of a quadrupole structure, for FWHM $=80 \mu \mathrm{m}$ an irregular dynamics is seen. For the next two examples $(\mathrm{FWHM}=90 \mu \mathrm{m}$ and $\mathrm{FWHM}=100 \mu \mathrm{m})$ a hexagonal structure is observed in the beginning, which is followed by an irregular oscillation of fragments in the first example and a regular rotation of fragments in the second example. Since for the parameters of such stable periodic solution $(\mathrm{FWHM}=100 \mu \mathrm{m})$ there exists no stable steady state, and since numerically Eq. (2) is equivalent to a scalar nonlinear delay differential equation, this phenomenon is recognized as a supercritical Hopf bifurcation. For FWHM $=110 \mu \mathrm{m}$ a stable quadrupole structure appears, whereas for FWHM $=115 \mu \mathrm{m}$ a quasistable transition from 


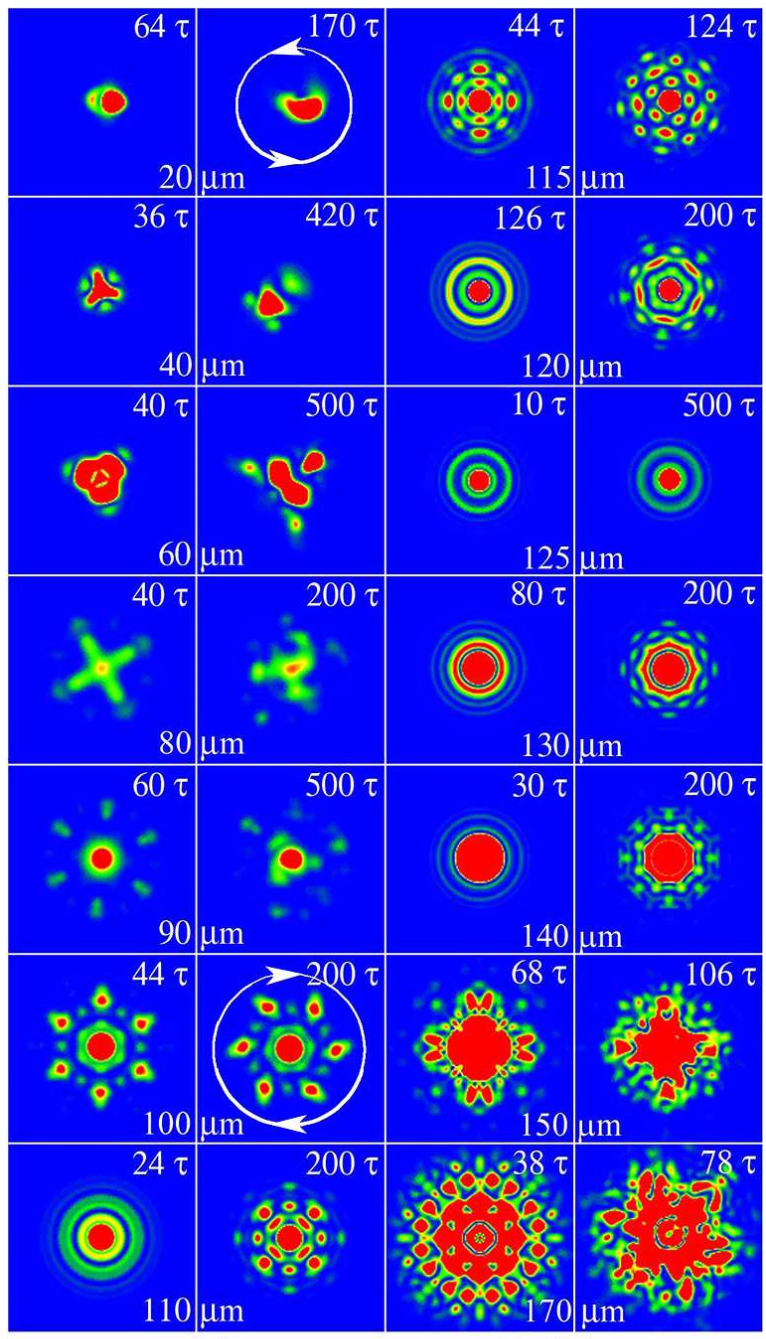

(a)

(b)

Fig. 1. Different dynamical structures seen in the forward beam, as its FWHM is increased, for the order parameter $A \Gamma L=2$. Each structure is presented at two instances. The figure should be viewed as an extended two-column picture, broken into (a) and (b) parts. For details, see the text. Other parameters: $\left|F_{0}\right|^{2}=\left|B_{L}\right|^{2}=3, \Gamma=10.89$, $L=3 L_{\mathrm{D}}$.

a quadrupole into a pentagon is seen. For FWHM $=120 \mu \mathrm{m}$ only a stable pentagonal structure is visible, but for FWHM $=125 \mu \mathrm{m}$ a stable necklace structure occurs. For FWHM $=130 \mu \mathrm{m}$ and $140 \mu \mathrm{m}$, two different stable octagonal structures appear. For the next two FWHM, after a set of various regular patterns, irregular structures take place. Even though the combination of parameters $A \Gamma L$ 
naturally appears in the threshold condition, the above-threshold behavior of the system evidently depends on the values of individual parameters.

Further, we investigate the interaction of CP Gaussian beams in fixed circular photonic lattices (CPL) with two ring defects (Fig. 2). We choose CPL with the first and third rings missing, and with the FWHM of input beams corresponding to the radii of the missing rings. We consider CP Gaussians with different widths (Fig. 2). We choose the forward beam to be narrower (FWHM corresponding to the radius of the first missing ring) than the backward beam (FWHM corresponding to the radius of the second missing ring). The general result is the appearance of different stable localized filamented structures with several parts, in the form of standing waves. For particular values of the control parameters $\Gamma$ and $L$ we observe stable dynamical structures, such as the rotating fivefold structure (the fourth row). Fivefold symmetry of the rotating structure would seem unexpected in view of the sevenfold symmetry of the lattice, however the general appearance of filaments follows the sequence threefold, fourfold, fivefold, sixfold, regardless of the symmetry of the lattice. The second ring of lattice beams shows the tendency of acting like a circular layer of lower refractive index. In all the cases considered, the narrower beam is strongly pinned to the central lattice site, whereas the wider one is more spread out.
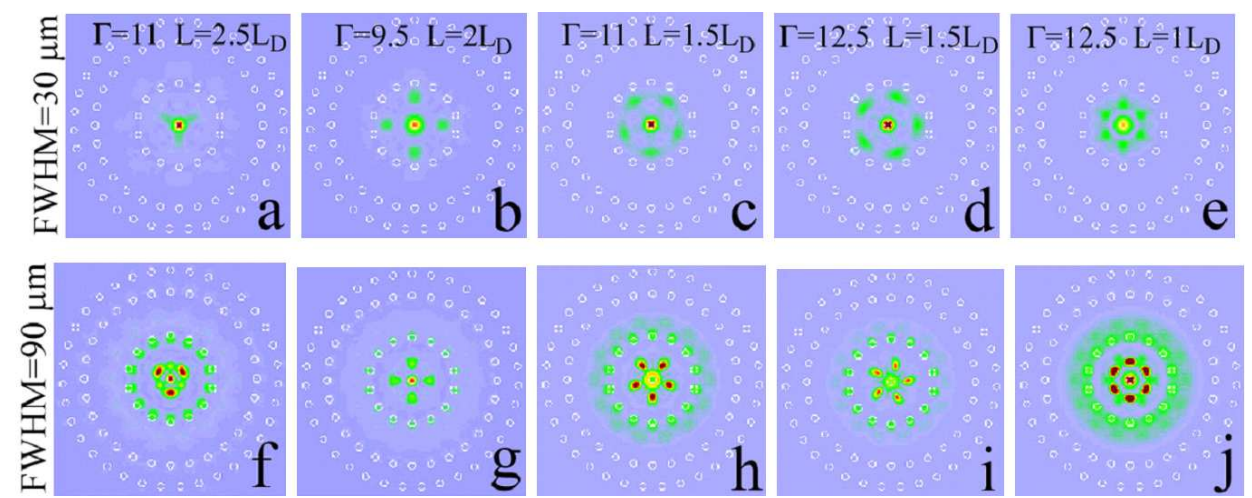

Fig. 2. Gaussian-Gaussian interaction of CP components with different widths in the 7-fold CPL with 2 ring defects. Intensity distributions of the forward beam with FWHM $=30 \mu \mathrm{m}$ (the first row) and the backward beam with FWHM $=90 \mu \mathrm{m}$ (the second row), for different values of the control parameters $\Gamma$ and $L$ are shown. For $\Gamma=12.5$ and $L=1.5 L_{\mathrm{D}}$, stable rotating structures, such as the rotating pentapole are observed (the fourth column). Other parameters: lattice spacing $30 \mu \mathrm{m}, \mathrm{FWHM}$ of the lattice beams $9 \mu \mathrm{m}$, maximum lattice intensity $I_{\mathrm{g}}=5 I_{d},\left|F_{0}\right|^{2}=\left|B_{L}\right|^{2}=1$.

Light propagating in a periodic dielectric medium, such as the photonic lattice, can behave similarly to electrons propagating in a crystalline potential, and can exhibit some of the fundamental quantum mechanical phenomena [4]. 


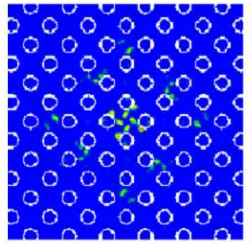

(a)

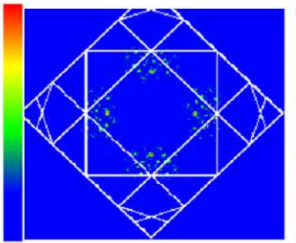

(b)

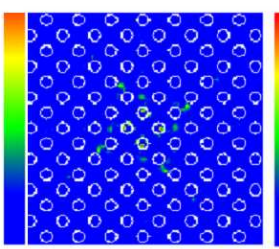

(c)

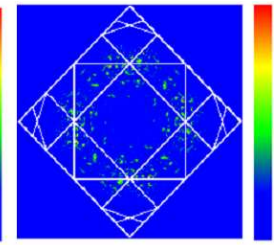

(d)

Fig. 3. Zener tunneling of two CP vortices with opposite topological charges \pm 2 , in the tilted square lattice. (a) and (c) The direct space. (b) and (d) The inverse space. Parameters: $\Gamma=16.6, L=4$ in (a) and $L=6$ in (c), FWHM of lattice beams $9 \mu \mathrm{m}$, lattice spacing $28 \mu \mathrm{m}, \mathrm{FWHM}$ of vortices $16.4 \mu \mathrm{m}$.

For CP beams in optically induced photonic lattices we observe oscillations of the beams inside the first Brillouin zone and tunneling of light from the first to the higher-order bands of the lattice band-gap spectrum. This effect, known as the Zener tunneling, is shown in Fig. 3.

Two wide $\mathrm{CP}$ vortices of opposite topological charges \pm 2 are launched head-on into a tilted square lattice, centered midway between the lattice sites. The figure depicts the exit face. At low values of $\Gamma$ and small thicknesses $L$ the beams remain confined within the first Brillouin zone. However, at a higher value of the coupling constant $\Gamma=16.6$ and for crystal thicknesses varied from $L=4$ in Fig. 3a to $L=6$ in Fig. 3c, a strong tunneling is found. Figures $3 \mathrm{~b}$ and $\mathrm{d}$ present the situation in the inverse space. As it is readily seen, the tunneling from the first Brillouin zone to the second (Fig. 3b), third and even fourth (Fig. 3d) Brillouin zones is observed.

In the end, we discuss the question of the conservation of angular momentum $(\mathrm{AM})$ in the interacting and fixed optically induced photonic lattices [5]. The conservation of AM in these systems is the consequence of the $\mathrm{O}(2)$ rotational symmetry in the transverse plane. As long as this symmetry is preserved during propagation, the individual momenta of $\mathrm{CP}$ beams, as well as the total AM, are conserved. However, our systems are so configured that their propagation is not stable, and their symmetry is not always $\mathrm{O}(2)$. We consider here two pairs of $\mathrm{CP}$ beams, a pair of lattices and a pair of vortices carrying unit charges of opposite sign (Fig. 4). The CP components propagate in the potential formed by the intensity distribution of the lattice beams, and vice versa. This propagation is unstable. As the vortices disintegrate, owing to modulational instabilities, their continuous symmetry is broken, and the law of AM conservation for them is no longer valid. Their AM starts to decrease, the difference being transferred to the lattices. Likewise for the lattices which propagate in the potential formed by the CP beams. As their potential starts to change, their AM starts to increase, the excess coming from the CP beams. The overall $\mathrm{O}(2)$ symmetry of the system is preserved, and the sum of AM remains nearly constant. But it is not strictly 


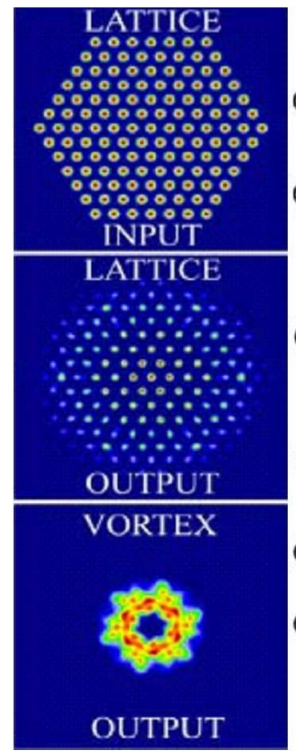

(a)

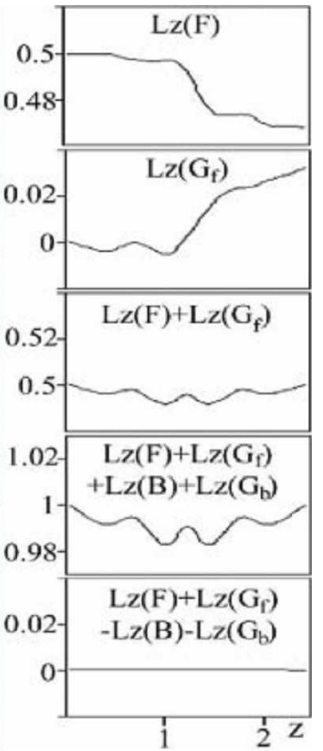

(b)

Fig. 4. Transfer of angular momentum in the interacting CP beams. (a) Forward lattice at the input and output faces, and the vortex at the output face. (b) Different normalized angular momenta. Parameters: $\Gamma=3, L=2.5 L_{\mathrm{D}}=10 \mathrm{~mm}$, lattice spacing $28 \mu \mathrm{m}$, FWHM of input vortices $24.6 \mu \mathrm{m}$, FWHM of input lattice beams $9 \mu \mathrm{m}$, maximum input intensity $\left|F_{0}\right|^{2}=\left|B_{L}\right|^{2}=5 I_{\mathrm{d}}$, and the maximum input lattice intensity $\left|G_{\mathrm{f}}\right|^{2}(z=0)$ $=\left|G_{\mathrm{b}}\right|^{2}(z=L)=20 I_{\mathrm{d}}$.

constant. However, when the difference of AM is calculated, it is found exactly constant. Hence, the conserved quantity for the CP beams is not the sum of AM, but the difference of AM. The conserved quantity for the CO beams is always the sum of AM.

The situation changes drastically for the fixed lattices (not shown). The transfer of AM is then precluded, and the law of AM conservation is no longer valid. The propagation of $\mathrm{CP}$ vortices proceeds in a discrete periodic potential, in this case of $C_{6 v}$ symmetry, and the $\mathrm{O}(2)$ symmetry is gone. AM is no longer a good "quantum number". A new conserved quantity must be introduced, the Bloch or pseudo AM.

\section{Conclusion}

A review of work on the dynamical behavior of CP mutually incoherent laser beams in PR crystals is presented. The development of patterns in broad hyper-Gaussian CP beams in saturable Kerr-like media is investigated, by varying the width of beams. Properties of CP self-trapped beams in optically induced circular photonic lattices with ring defects are investigated numerically. The existence of 
various phenomena characteristic of solid state physics, such as Bloch oscillations, Zener tunneling, and Anderson localization are demonstrated in the propagation of $\mathrm{CP}$ beams in photonic lattices. While the total AM of copropagating beams is always conserved, that of $\mathrm{CP}$ beams is not. The difference of AM of CP beams is conserved, not the sum. In the fixed lattices there is always considerable loss of AM.

\section{References}

[1] M. Haelterman, A.P. Sheppard, A.W. Snyder, Opt. Commun. 103, 145 (1993); O. Cohen, S. Lan, T. Carmon, Opt. Lett. 27, 2013 (2002); M. Belić, Ph. Jander, A. Strinić, A. Desyatnikov, C. Denz, Phys. Rev. E 68, 025601(R) (2003).

[2] M. Belić, M. Petrović, D. Jović, A. Strinić, D. Arsenović, K. Motzek, F. Kaiser, Ph. Jander, C. Denz, M. Tlidi, P. Mandel, Opt. Express 12, 708 (2004); D. Jović, D. Arsenović, A. Strinić, M. Belić, M. Petrović, Opt. Express 13, 4382 (2005); D. Jović, M. Petrović, M. Belić, J. Schroeder, Ph. Jander, C. Denz, Opt. Express 13, 10717 (2005); M. Petrović, D. Jović, M. Belić, J. Schröder, Ph. Jander, C. Denz, Phys. Rev. Lett. 95, 053901 (2005).

[3] C. Denz, M. Schwab, C. Weilnau, Transverse Pattern Formation in Photorefractive Optics, Springer, Berlin 2003.

[4] H. Trompeter, W. Krolikowski, D.N. Neshev, A.S. Desyatnikov, A.A. Sukhorukov, Y.S. Kivshar, T. Pertsch, U. Peschel, F. Lederer, Phys. Rev. Lett. 96, 053903 (2006); T. Schwartz, G. Bartal, S. Fishman, M. Segev, Nature 446, 52 (2007).

[5] M.S. Petrović, D.M. Jović, S. Prvanović, M.R. Belić, Phys. Rev. A 76, 023820 (2007) 